
https://doi.org/10.30534/ijeter/2021/16972021

\title{
Determination of the Optimal Parameters in Brass Turning Process by Taguchi method
}

\author{
B. Surapong ${ }^{1}$, B. Phiraphan ${ }^{2}$, B. Wiboonluk ${ }^{3}$ \\ ${ }^{1}$ Department of Industrial Technology Education, Faculty of Engineering, Rajamangala University of Technology \\ Lanna, Thailand, pong_pang49@yahoo.com, surapongb@edu.rmutl.ac.th \\ ${ }^{2}$ Department of Industrial Technology Education, Faculty of Engineering, Rajamangala University of Technology \\ Lanna, Thailand, foundry18@yahoo.com \\ ${ }^{3}$ Department of Student development Division, Rajamangala University of Technology Lanna. 128 Huay kaew \\ Road, Muang District, Chiang Mai 50300, Thailand, Viboonluk_bp@ hotmail.com
}

\begin{abstract}
This research is a study of the turning process by testing with brass material. There are three control factors: spindle of speed, feed rate, and depth of cut respectively. The turning process requires variable control, affect the quality of production productivity and production cost planning an experiment with the Taguchi Method help in the experiment the analysis of variance, orthogonal array, and signal and noise ratios were considered as an experiment and survey of brass turning characteristics to determine the lowest material removal rate. The results obtained from the experiment were used to repeat the experiment for confirmation. This requires the turning process to be reliable and optimized.
\end{abstract}

Key words: Optimal, parameter, ANOVA, regression of coefficient, optimization, turning process, taguchi method

\section{INTRODUCTION}

Lathes are basic machines used to manufacture parts and equipment in industrial plants. And educational institutions in various universities, therefore, lathes are very necessary for the production or processing of various materials. Therefore, the operation steps in the turning process there are many factors involved such as lathe machine, cutting tool, turning knife angle, turning tool material, coolant, workers must be competent and skilled in the use of the lathe, speed, feed rate, depth of cut, and the materials used for production.

Taguchi [1], [2] design of experiment (DOE) methods incorporate fractional factorial matrixes or orthogonal arrays to minimize the number of experiments required to achieve a given set of performance characteristics. Iterative Taguchi experiments can be designed to systematically approach optimal parameters for a complicated process or as quality assurance tool to identify the important parameters to monitor for Statistical Process Control (SPC). The Taguchi experimental approach allows a statistically sound experiment to be completed, while investigating a minimum number of possible combinations of parameters or factors. A Taguchi experiment can be accomplished in a timely manner and at a reduced cost with results comparable to a full factorial experiment. Used design of experiments concepts to optimize the process parameters of chemical-mechanical polishing process in wafer manufacturing. Material removal rate and non-uniformity of surface profiles were selected as the target values, and the process parameters such as platen speeds, carrier speeds, back side pressure, slurry flow rate and a head down force were optimized [3]. Conducted experiments to know the influence of forming process parameters with an objective to minimize the load on the tool [4]. The process parameters such as strain, die angle and coefficient of friction were optimized by using both classical optimization techniques and design of experiments concepts. The merits and demerits of both the classical and DOE methods were highlighted. In this study, the authors calculated the maximum expansion ratio of the tube and the fracture behavior of the tube. The corresponding changes in the hardness values were investigated. Studied the influence of process parameters on the deep drawing of stainless steel. Deep drawing process parameters such as die radius, blank holder force and friction-coefficient were studied to find the optimum condition for forming. It was found that the die radius followed by the blank holder force and friction coefficient have the greatest influence on the deep drawing of stainless steel blank [5]. Optimized aluminium foam manufacturing process with Taguchi method. In this study, the relative density of aluminium foams were minimized by optimally controlling three process parameters namely silicon carbide content in powder mixture, the compaction pressure and the forming temperature [6]. Applied the artificial neural networks (ANN) to predict the forming load of magnesium alloy under hot extrusion. In this study, ANN analysis was used to determine the die shapes for various extrusion ratios [7]. Implemented multi-objective 
B. Surapong et al., International Journal of Emerging Trends in Engineering Research, 9(7), July 2021, 929 - 936

optimization to optimize the gear warm forming process parameters [8].

The main factors in lathe production include turning, cutting fluid, coolant, rpm, cutting speed, feed rate, depth of cut operating environment. Operator skills, expertise, and experience are extremely important. As well as production materials, material removal rate.

In this research, the effects of the turning process were studied by testing on brass material. The material is a manufacturing component that is essential in the manufacture of any machine. The Taguchi Method experiment was designed to determine the lowest possible material removal rate per production process, check out the orthogonal array, noise and signal ratio, and the analysis of variance under a significant statistical assumption.

\section{LITERATURE REVIEW}

T. Tamizharasan et. al. [9] had developed a mathematical model using multiple regression analysis and artificial neural network (ANN) model for artificial intelligent model. They found that depth of cut is most significant parameter followed by cutting speed, hardness of material and lastly feed rate. The mathematical model developed by multiple regression method shows accuracy of $97.41 \%$ which is reliable to be used on AE signal prediction. The result from this research is useful to be implemented in industry to reduce time and cost in $\mathrm{AE}$ signal prediction.

R S Pawade et. al. [10] has found that analysis of AE Signal during the machining could help to determine the quality of the machine surface. Frequency amplitude of the AE signal is influence by the cutting speed. The feed rate and edge geometry are found to influence the number of count generated during machining deformation.

T S Reddy et. al. [11] have done the turning operation using HSS tool on mild steel. The result shows significance relation between surface roughness and $\mathrm{AE}$ signals parameters. The conclusions are made for predicting surface roughness by suggesting consistence values and ranges for on-line monitoring of $\mathrm{AE}$ signals parameters.

J Bhaskaran et. al. [12] in study of hard turning, used the skew and kurtosis parameters of AE signals to monitor tool wear. The moment parameters of AErms signals such as skew and kurtosis can be used to reliably monitor the tool wear and surface roughness.

G. Priyadarshini et. al. [13] found that lower feed rate/rev, AErms Value was less and surface roughness of machined surface was also less. Analysis reveal that the acoustic emission while metal machining can be very useful in identifying relative surface quality of the product and hence selection of proper cutting parameters. Acoustic emission technique can be effectively used in online condition monitoring of machining parameters.

Nitin Sharma et. al. [14] have used Taguchi method to find the optimal cutting parameters for surface roughness (Ra) in turning of AISI-410 steel bars usinf tin coated inserts. The four cutting parameters namely insert radius, depth of cut, feed and cutting speeds are optimized with consideration of surface roughness. The analysis reveals that feed rate has the most significant effect on Ra. They found that parameter design of the Taguchi method provides a simple systematic and efficient methodology for the optimization of the cutting parameters.

Suleman et. al. [15] have investigated the influence of depth of cut, feed rate and spindle speed on surface roughness during turning of mild steel. Analysis of variance (ANOVA) is used to analyze the influence of machining parameters on surface roughness. Multiple linear regression, mathematical model correlating the influence of machining parameters on surface roughness during the process were developed and validated.

Prof. Atul dhale1 et. Al [16] use as a technique for quantitative studies of manufacturing processes. Optimum selection of cutting conditions importantly contribute to the increase of productivity and the reduction of cost, therefore utmost attention is paid to this problem in this contribution. In this paper we proposed $\mathrm{AE}$ as non-contact and indirect technique for in-process surface roughness assessment in turning. Three cutting conditions dry cut, cutting with water as coolant and normal coolant were used. The material used in study is EN8. Three cutting parameters namely feed rate, depth of cut, cutting speed are optimized with consideration with surface roughness.

\section{TAGUCHI METHOD}

Taguchi's philosophy, developed by Dr. Genichi Taguchi, is an efficient tool for the design of high quality manufacturing system. Taguchi's Orthogonal Array (OA) provides a set of well-balanced experiments (with less number of experimental runs), and Taguchi's signal-to-noise ratios $(\mathrm{S} / \mathrm{N})$, which are logarithmic functions of desired output; serve as objective functions in the optimization process. Taguchi method uses a statistical measure of performance called signal-to-noise ratio. The $\mathrm{S} / \mathrm{N}$ ratio takes both the mean and the variability into account. The $\mathrm{S} / \mathrm{N}$ ratio is the ratio of the mean (Signal) to the standard deviation (Noise). The ratio depends on the quality characteristics of the product/process to be optimized. The standard $\mathrm{S} / \mathrm{N}$ ratios generally used are as follows: - 
Nominal-is-Best (NB), lower-the-better (LB) and Higher-the-Better (HB). The optimal setting is the parameter combination, which has the highest $\mathrm{S} / \mathrm{N}$ ratio, [17], [18]. Because, irrespective of the quality criteria may be (NB, LB, $\mathrm{HB}) \mathrm{S} / \mathrm{N}$ ratio should always be maximized. Once experimental data (quality attribute value) is normalized using $\mathrm{NB} / \mathrm{LB} / \mathrm{HB}$ criteria; normalized value lies in between zero to one. Zero represents worst quality to be rejected and one represents most satisfactory quality. Since $\mathrm{S} / \mathrm{N}$ ratio is expressed as mean (signal) to the noise (deviation from the target); maximizing $\mathrm{S} / \mathrm{N}$ ratio ensures minimum deviation and hence it is ( $\mathrm{S} / \mathrm{N}$ ratio) to be maximized [19].

Experimental design by various Taguchi methods, Taguchi experimental design offers distinct advantages by which many factors can be examined simultaneously and much quantitative information can be extracted with a few experimental trials [20], [21]. A few reports are available on the application of Taguchi's method in the field of biotechnology [22]-[24]. The basic principle of this method serves as screening filters which examine the effects of many process variables and identify those factors which have major effects on process using a few experiments [25]. Taguchi method of DOE involves the establishment of a large number of the experimental situation described as orthogonal arrays (OA) to reduce experimental errors and to enhance their efficiency and reproducibility of the laboratory experiments or workshops in university but operation of control under the hypothesis of the experimental according [26].

\section{EXPERIMENTAL DESIGN}

Experiments will be conducted on a selected lathe, selected tools and materials were carbides the three process parameters as stated above. Spindle of speed (A), feed rate (B) and depth of cut (C) were considered in the study. Spaced five evenly levels within the operating range of the input parameters were selected for each process parameter. By experimenting with the Taguchi method, A L27 (OA) orthogonal array has been developed that includes 27 different experiments at three levels. According [27] the Taguchi method was selected to obtain the most suitable solution for material removal rates in a given input range such as rake angle, cutting speed and feed.

Therefore, modern methods of finding the best results above a given set of inputs can be easily accomplished using the Taguchi method rather than the other conventional methods. This method has a wide application scope in various fields of engineering science. The experimental data were analyzed using Minitab Release 19.00 software. To overcome this problem [28] Taguchi suggested a specially designed method called the use of orthogonal array to study the entire parameter space with lesser number of experiments to be conducted. Taguchi thus, recommends the use of the loss function to measure the performance characteristics that are deviating from the desired target value. The value of this loss function is further transformed into signal-to-noise $(\mathrm{S} / \mathrm{N})$ ratio. Usually, there are three categories of the performance characteristics to analyze the $\mathrm{S} / \mathrm{N}$ ratio. They are: nominal-the-best, larger-the-better, and smaller-the-better.

\subsection{Steps Involved in Taguchi Method}

The use of Taguchi's parameter design involves the following steps [29].

a. Identify the main function and its side effects.

b. Identify the noise factors, testing condition and quality characteristics.

c. Identify the objective function to be optimized.

d. Identify the control factors and their levels.

e. Select a suitable orthogonal array and construct the matrix

f. Conduct the matrix experiment.

g. Examine the data; predict the optimum control factor levels and its performance.

h. Conduct the verification experiment.

\subsubsection{The process of preparing for efficiency}

The process of preparing equipment and materials to be tested for efficiency takes the principles of a Taguchi experiment design to determine the optimization in the manufacturing process for lathes. The testing procedure of the machining process for the best value by the Taguchi method is shown in Figure 1 and Table 1 shows the design factors along with their level.

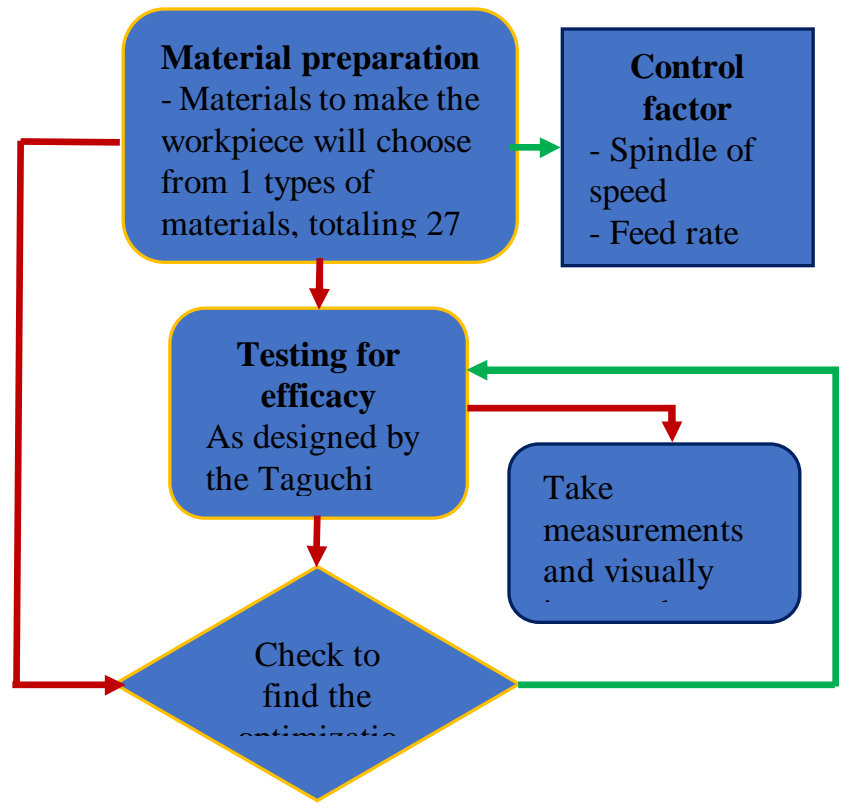

Figure 1: The testing process of the machining process for the optimization by the Taguchi method 
B. Surapong et al., International Journal of Emerging Trends in Engineering Research, 9(7), July 2021, 929 - 936

\subsubsection{The objective function}

Objective function: Smaller-the-Better

S/N Ratio for this function:

$S / N_{S}=-10 \log \left(\frac{1}{n} \sum_{i=1}^{n} y_{i}^{2}\right)$

Where, $\mathrm{n}=$ Sample Size, and $\mathrm{y}=\mathrm{MRR}$ in that run.

\subsubsection{The control factors and parameters used in the experiment}

The factors and parameters used in the experiment were determined in the conduct of the experiment and based on the guidelines outlined in the manufacturer's turning operation. In this study spindle of speed, flow rate and depth of cut was selected as control factors and their levels are shown in (see Table 1).

Table 1: Factors and parameters used in the experiment

\begin{tabular}{|l|c|c|c|}
\hline Level & $\begin{array}{c}\text { Spindle of } \\
\text { speed }(\mathrm{rpm})\end{array}$ & $\begin{array}{c}\text { Flow rate } \\
(\mathrm{mm} / \mathrm{rev})\end{array}$ & $\begin{array}{c}\text { Depth of cut } \\
(\mathrm{mm} .)\end{array}$ \\
\hline 1 & 200 & 0.25 & 0.3 \\
\hline 2 & 220 & 0.36 & 0.5 \\
\hline 3 & 270 & 0.45 & 0.7 \\
\hline
\end{tabular}

\subsubsection{Selection of orthogonal array}

To select an appropriate orthogonal array for conducting the experiments, the degrees of freedom are to be computed. The same is given below:

Degrees of Freedom: 1 for mean value, and $8=(2 \times 4)$, two each for the remaining factors

Total degrees of freedom: 9

The most suitable orthogonal array for experimentation is L27 array as shown in (see Table 2). Therefore, a total twenty-seven experiments are to be carried out.

Table 2: Orthogonal array (OA) L27

\begin{tabular}{|l|l|l|l|}
\hline \multirow{2}{*}{ Experiment No. } & \multicolumn{3}{|c|}{ Control factors } \\
\cline { 2 - 4 } & 1 & 2 & 3 \\
\hline 1 & 1 & 1 & 1 \\
\hline 2 & 1 & 1 & 1 \\
\hline 3 & 1 & 1 & 1 \\
\hline 4 & 1 & 2 & 2 \\
\hline 5 & 1 & 2 & 2 \\
\hline 6 & 1 & 2 & 2 \\
\hline 7 & 1 & 3 & 3 \\
\hline 8 & 1 & 3 & 3 \\
\hline 9 & 1 & 3 & 3 \\
\hline 10 & 2 & 1 & 2 \\
\hline 11 & 2 & 1 & 2 \\
\hline
\end{tabular}

Table 2: (cont.) Orthogonal array (OA) L27

\begin{tabular}{|l|l|l|l|}
\hline \multirow{2}{*}{ Experiment No. } & \multicolumn{3}{|c|}{ Control factors } \\
\cline { 2 - 4 } & 1 & 2 & 3 \\
\hline 12 & 2 & 1 & 2 \\
\hline 13 & 2 & 2 & 3 \\
\hline 14 & 2 & 2 & 3 \\
\hline 15 & 2 & 2 & 3 \\
\hline 16 & 2 & 3 & 1 \\
\hline 16 & 2 & 3 & 1 \\
\hline 17 & 2 & 3 & 1 \\
\hline 18 & 2 & 3 & 1 \\
\hline 19 & 3 & 1 & 3 \\
\hline 20 & 3 & 1 & 3 \\
\hline 21 & 3 & 1 & 3 \\
\hline 22 & 3 & 2 & 1 \\
\hline 23 & 3 & 2 & 1 \\
\hline 24 & 3 & 2 & 1 \\
\hline 25 & 3 & 3 & 2 \\
\hline 26 & 3 & 3 & 2 \\
\hline 27 & & 3 & 2 \\
\hline
\end{tabular}

\subsubsection{Analysis of Variance (ANOVA)}

ANOVA is a statistical tool used to test differences between two or more means. It may seem odd that the technique is called analysis of variance (ANOVA) rather than analysis of means. The analysis of variance is obtained by dividing the measured the sum of the squared deviations from the total mean $\mathrm{S} / \mathrm{N}$ ratio into contributions by each of the control factors and the errors. [30] Analysis of variance identify the percentage of contribution of controlled process parameters.

\section{ANALYSIS OF EXPERIMENTAL RESULTS}

\subsection{Results}

Table 3 shows the experiment results for the MRR and corresponding $\mathrm{S} / \mathrm{N}$ ratios were obtained with the help of Minitab release19.00 software.

\subsection{Cause of spindle of speed, flow rate and depth of cut on MRR}

From the response in (see Table 3), it is clear that cutting speed is the most influencing factor followed by spindle of speed, depth of cut, and flow rate for MRR. The optimum for MRR is a spindle of the speed of $220 \mathrm{rpm}$, a flow rate of 0.25 $\mathrm{mm} / \mathrm{rev}$, and a depth of cut of $0.5 \mathrm{~mm}$ respectively.

\subsection{Material removal rate measurement}

The Material removal rate is employed to work out the quantity of material removed per second. It's given by the formula

MRR = SS FR DC 
B. Surapong et al., International Journal of Emerging Trends in Engineering Research, 9(7), July 2021, 929 - 936

Where SS $=$ Spindle of speed $(\mathrm{rpm})$

$\mathrm{FL}=$ Flow rate $(\mathrm{mm} / \mathrm{rev})$

$\mathrm{DC}=$ Depth of cut $(\mathrm{mm})$

MRR $=$ Material removal rate $(\mathrm{mm} 3 / \mathrm{min})$

As the conditions for feed, cutting rate and depth of cut area unit mounted therefore, this formula is employed to calculate the MRR rather than hard the initial and also the final weight, the higher than formula was won't to calculate the MRR [31].

Table 3: Factors and parameters used in the experiment

\begin{tabular}{|c|c|c|c|c|}
\hline Level & $\begin{array}{c}\text { Spindle of } \\
\text { speed } \\
(\mathrm{rpm})\end{array}$ & $\begin{array}{l}\text { Flow rate } \\
(\mathrm{mm} / \mathrm{rev})\end{array}$ & $\begin{array}{l}\text { Depth of } \\
\text { cut (mm.) }\end{array}$ & \multirow[t]{2}{*}{ MRR } \\
\hline N0 & $\mathrm{SS}$ & FL & $\mathrm{DC}$ & \\
\hline 1 & SS & FL & $\mathrm{DC}$ & MRR \\
\hline 2 & 200 & 0.25 & 0.3 & 4.638 \\
\hline 3 & 200 & 0.25 & 0.3 & 4.621 \\
\hline 4 & 200 & 0.25 & 0.3 & 4.625 \\
\hline 5 & 200 & 0.36 & 0.5 & 11.933 \\
\hline 6 & 200 & 0.36 & 0.5 & 11.820 \\
\hline 7 & 200 & 0.36 & 0.5 & 11.905 \\
\hline 8 & 200 & 0.45 & 0.7 & 13.333 \\
\hline 9 & 200 & 0.45 & 0.7 & 13.193 \\
\hline 10 & 200 & 0.45 & 0.7 & 13.193 \\
\hline 11 & 220 & 0.25 & 0.5 & 4.888 \\
\hline 12 & 220 & 0.25 & 0.5 & 4.864 \\
\hline 13 & 220 & 0.25 & 0.5 & 4.840 \\
\hline 14 & 220 & 0.36 & 0.7 & 12.853 \\
\hline 15 & 220 & 0.36 & 0.7 & 12.788 \\
\hline 16 & 220 & 0.36 & 0.7 & 12.788 \\
\hline 17 & 220 & 0.45 & 0.3 & 14.493 \\
\hline 18 & 220 & 0.45 & 0.3 & 13.966 \\
\hline 19 & 220 & 0.45 & 0.3 & 14.368 \\
\hline 20 & 270 & 0.25 & 0.7 & 9.653 \\
\hline 21 & 270 & 0.25 & 0.7 & 9.506 \\
\hline 22 & 270 & 0.25 & 0.7 & 9.560 \\
\hline 23 & 270 & 0.36 & 0.3 & 15.773 \\
\hline 24 & 270 & 0.36 & 0.3 & 15.480 \\
\hline 25 & 270 & 0.36 & 0.3 & 15.337 \\
\hline 26 & 270 & 0.45 & 0.5 & 17.065 \\
\hline 27 & 270 & 0.45 & 0.5 & 17.182 \\
\hline
\end{tabular}

\subsection{Analysis of signal to noise ratio and means}

Smaller is better performance characteristic was selected to obtain material removal rate.

Table 4: Response Table for signal to noise ratios (Smaller is better)

\begin{tabular}{|l|c|c|c|}
\hline \multicolumn{1}{|c|}{ Level } & SS & FL & DC \\
\hline 1 & -19.08 & -15.56 & -20.08 \\
\hline 2 & -19.66 & -22.49 & -19.95 \\
\hline 3 & -22.69 & -23.38 & -21.40 \\
\hline Delta & 3.61 & 7.83 & 1.45 \\
\hline Rank & 1 & 2 & 3 \\
\hline
\end{tabular}

Table 5: Response Table for means

\begin{tabular}{|l|c|c|c|}
\hline \multicolumn{1}{|c|}{ Level } & SS & FL & DC \\
\hline 1 & 9.918 & 6.355 & 11.478 \\
\hline 2 & 10.650 & 13.409 & 11.259 \\
\hline 3 & 14.043 & 14.848 & 11.874 \\
\hline Delta & 4.125 & 8.493 & 0.615 \\
\hline Rank & 1 & 2 & 3 \\
\hline
\end{tabular}

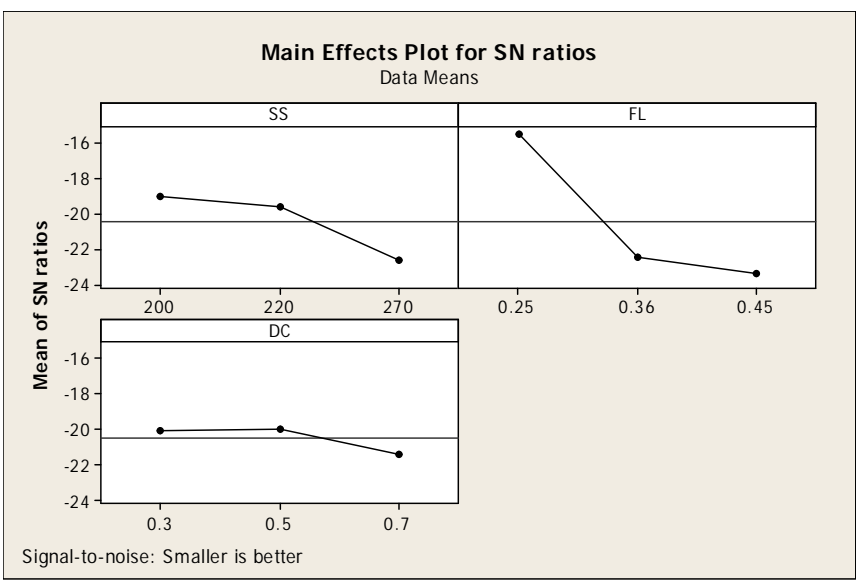

Figure 2: Main effects plot for $\mathrm{SN}$ ratios

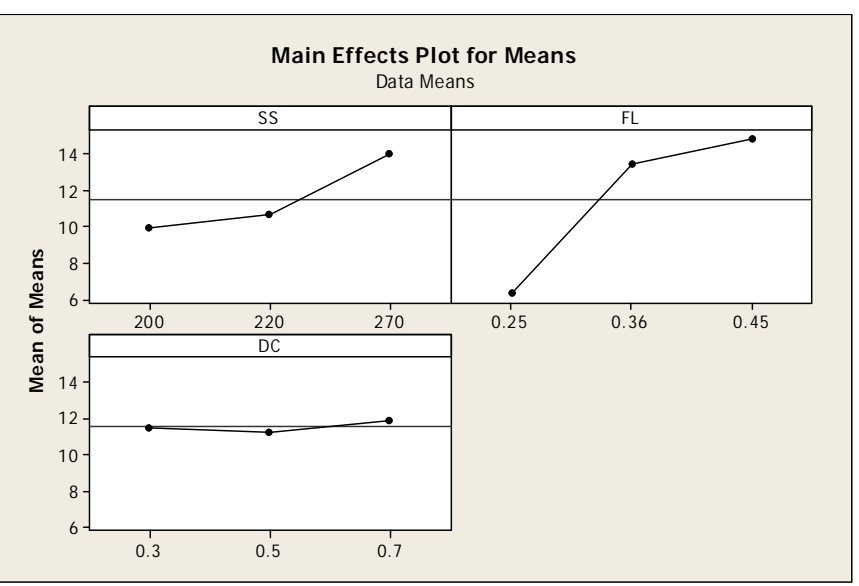

Figure 2: Main effects plot for means

From Tables 4 and 5, and Figure 2 and 3 above that flow and edge condition after that spindle of speed plays an important role for MRR, a spindle of speed $=220 \mathrm{rpm}$, feed rate $=0.25 \mathrm{~mm} / \mathrm{rev}$ and depth of cut $=0.30 \mathrm{~mm}$ respectively.

\subsection{Analysis of variance (ANOVA) and estimated model coefficients}

Minitab release 19.00 was used to investigate the process parameters. Analysis of variance and estimate coefficient defines the percentage of contribution for each process parameters. 
B. Surapong et al., International Journal of Emerging Trends in Engineering Research, 9(7), July 2021, 929 - 936

Table 6: Analysis of variance for means

\begin{tabular}{|l|c|l|l|l|l|l|}
\hline Source & DF & Seq SS & Adj SS & MS & F & \multicolumn{1}{c|}{ P } \\
\hline SS & 2 & 29.072 & $\begin{array}{l}29.072 \\
29.072\end{array}$ & 14.5360 & 38.95 & $\begin{array}{l}0.02 \\
5\end{array}$ \\
\hline FR & 2 & $\begin{array}{l}123.94 \\
7\end{array}$ & $\begin{array}{l}123.94 \\
7\end{array}$ & 61.9736 & $\begin{array}{l}166.0 \\
5\end{array}$ & $\begin{array}{l}0.00 \\
6\end{array}$ \\
\hline DC & 2 & 0.583 & 0.583 & 0.2914 & 0.78 & $\begin{array}{l}0.56 \\
2\end{array}$ \\
\hline $\begin{array}{l}\text { Residua } \\
\text { l Error }\end{array}$ & 2 & 0.746 & 0.746 & 0.3732 & & \\
\hline Total & 8 & $\begin{array}{l}154.34 \\
8\end{array}$ & & & & \\
\hline
\end{tabular}

Table 7: Estimated model coefficients for means

\begin{tabular}{|l|c|c|c|c|}
\hline \multicolumn{1}{|c|}{ Term } & Coef & SE Coef & T & P \\
\hline Constant & 11.5370 & 0.2036 & 56.655 & 0.000 \\
\hline SS 200 & -1.6191 & 0.2880 & -5.622 & 0.030 \\
\hline SS 220 & -0.8873 & 0.2880 & -3.081 & 0.091 \\
\hline FL 0.25 & -5.1821 & 0.2880 & -17.994 & 0.003 \\
\hline FL 0.36 & 1.8716 & 0.2880 & 6.499 & 0.023 \\
\hline DC 0.3 & -0.0590 & 0.2880 & -0.205 & 0.857 \\
\hline DC 0.5 & -0.2779 & 0.2880 & -0.965 & 0.436 \\
\hline S =0.6109 & \multicolumn{5}{|c|}{ R-Sq $=99.5 \%$} & R-Sq(adj) $=98.1 \%$ \\
\hline
\end{tabular}

\subsection{ANOVA for material removal rate (MRR)}

Results obtained for the material removal rate (MRR) square measure shown in (see Table 3). The results for material removal rate (MRR) were obtained from the nine experiments performed of Taguchi. The experimental results analyzed with multivariate analysis square measure shown in (see Table 6). The $F$ value calculated through Minitab release 19.00 computer code is shown within the second last column of multivariate analysis table that suggests the importance of the factors on the required characteristics. Larger is that the F worth higher is that the significance (considering confidence level of 95\%). The results show that solely feed is that the most vital issue. The results are consistent with the research of [32].

\subsection{Signal -to - noise ratio}

Parameters that effect on the output are often divided into 2 parts: governable (or design) factors and uncontrollable (or noise) factors. The value of governable factors is often adjusted by the designer however the worth of uncontrollable factors can't be modified as a result of they're the sources of variation as a result of operational surroundings. The most effective set of management factors as they influence the output is set by performing arts experiments. Smaller-the-Better is employed for material removal rate shown in Figure 2 and 3 respectively. The values were consistent with the research of [32].

\subsection{Regression equation}

The regression equation for material removal rate as shown by equation (1) is calculated in Minitab release 19.00 software. The regression equation shows the significant effect of each process parameter on the material removal rate. From this equation, it is cleared that the current and pulse on time have positively affected the material removal rate whereas spindle of speed, feed rate, and depth of cut effect on response i.e. material removal rate.

\section{MRR}

$$
\begin{aligned}
& =11.5370(\text { constant })+(-1.6191)(\text { SS200 })+(-0.8873)(\text { SS } 220) \\
& +(-5.1821)(\text { FL0.25) }+(1.8716)(\text { FL } 0.36)+(-0.05890)(\text { DC0.3 }) \\
& +(-0.2779)(\text { DC } 0.5)
\end{aligned}
$$

\section{CONCLUSION AND FURTHER RESEARCH}

From the Experimental results as we observed that the optimum condition for our input data to get maximum material removal rate (MRR) is 9 degrees of the spindle of the speed of $220 \mathrm{rpm}, 0.25 \mathrm{~mm} / \mathrm{rev}$ of flow rate, and depth of cut of $0.3 \mathrm{~mm}$. The maximum material removal rate that we get from our experiment and the Taguchi method is 4.621 grams. The minimum material removal rate that we get from our experiment by the Taguchi method is 4.621 grams. This concludes that spindle of speed, flow rate, and depth of cut is consumed and from analysis, we got the minimum material removal rate conditions which would directly save power in small-scale productions. This would also save the lead time in the industrial production process.

Taguchi method is one method that has been used in research to create confidence and reliability in the production process significantly under statistical experimental assumptions.

In the following research, the experimental level has been increased to five experimental levels, five variables, and surface roughness. Because brass materials are used to make materials in milling machines, turning machines, etc., because the lowest material removal rate is needed to optimize the value. Next, find the appropriate surface roughness. And in further research or in the future, the cause and effect diagram principle will be used to help find factors that may affect the production process, such as people, machines, materials, methods, measurements, etc.

\section{ACKNOWLEDGEMENTS}

Financial support Department of Industrial Technology Education, Faculty of Engineering RMUTL: Rajamangala University of Technology Lanna, Chiang Mai, Thailand is gratefully acknowledged. 
B. Surapong et al., International Journal of Emerging Trends in Engineering Research, 9(7), July 2021, 929 - 936

\section{REFERENCES}

1. Phillip J. Ross. Taguchi techniques for quality engineering, McGraw-Hill, Inc., (1988).

2. Genichi Taguchi. Introduction to Quality engineering Engineering, Asian Productivity Organization, 1986.

3. Hsin-Te L, Jie-Ren S and Yung-Kuang Y. Applications of Taguchi and design of experiments methods in optimization of chemical mechanical polishing process parameters, International Journal of Advanced Manufacturing Technology, 2008; 38: 674 - 682.

4. Jurkovic Z, Jurkovic M and Buljan S. Optimization of extrusion force prediction model using different techniques, Journal of Achievements in Materials and Manufacturing Engineering, 2006; 17: 353 - 356.

5. Padmanabhan R, Oliveira MC, Alves JL and Menezes LF. Influence of process parameters on the deep drawing of stainless steel, Finite Elements in Analysis and Design, 2007; 43: 1062 - 1067.

6. Rossella S, Filippis LAC, Ludovico AD and Boghetich G. Application of Taguchi method for the multi objective optimization of aluminium foam manufacturing parameters, International Journal of Material Forming, 2010; 3: 1 - 5.

7. Hsiang SH and Jer-Liang K. Applying ANN to predict the forming load and mechanical property of magnesium alloy under hot extrusion, International Journal of Advanced Manufacturing Technology, 2005; 26: 970 - 977.

8. Wei $\mathrm{F}$ and Lin $\mathrm{H}$. Multi-objective optimization of process parameters for the helical gear precision forging by using Taguchi method, Journal of Mechanical Science and Technology, 2011; 25: 519 1527.

9. T. Tamizharasan, Kingston Barnabas. Prediction of acoustic emission in turning using multiple regression analysis and artificial neural network, Journal of Material Science, Vol. 1,pp 15-23,

10. R S Pawade, S S Joshi. Analysis of acoustic emission signals and surface integrity in the high speed turning of inconel 718, Journal of Engineering Manufacture Sage Journal, 2011,

11. T S Reddy, C E Reddy. Real time monitoring of surface roughness by acoustic emission in CNC turning, Journal of engineering science and technology review, 2010, volume 3 issue 1 .

12. J Bhaskaran, M. Murugan, N Balashnmugam and Chellamalai; Monitiring of hard turning using acoustic emission signal, Journal of material science and technology, Springer,2012, 26, pp. 609-615

13. G. Priyadarshini, Manpreet Bains b, Bhuvnesh Bhardwaj , P. K. Singh and Rajesh Kumar. Application of acoustic emission technique in surface tecture monitoring during turning operation, National Conference on Advancements and Futuristic Trends in Mechanical and Materials Engineering, February 19-20, 2010.
14. Nitin Sharma, Shahzad Ahmad, Zahid A. Khan, Arshad Noor Siddiquee, Optimization of cutting parameters for surface roughness in turning, International Journal of Advanced Research in Engineering and Technology (IJARET), ISSN 0976 - 6480(Print), ISSN 0976 6499(Online), 2012, Volume 3, Number 1, January June.

15. Suleiman Abdulkareem1, Usman Jibrin Rumah and Apasi Adaokoma, Optimizing machining parameters during turning process, International Journal of Integrated Engineering, Vol. 3 No. 1 (2011) p. 23-27

16. Prof. Atul dhale1 et. Al. Optimization by taguchi method and in process Monitoring of cutting parameters using acoustic emission for EN8, International Journal of Application or Innovation in Engineering \& Management (IJAIEM), 2013, Volume 2, Issue 11, November, pp.465-471.

17. Taguchi G. Introduction to quality engineering, proceedings of asian productivity organization, UNIPUB, White Plains, NY, 1986.

18. Mahapatra S.S. and Chaturvedi, V. Modeling and analysis of abrasive wear performance of composites using Taguchi approach, International Journal of Engineering, Science and Technology, 2009, Vol. 1, No. 1, pp. 123-135.

19. Sanjit Moshat, Saurav Datta, Asish Bandyopadhyay and Pradip Kumar Pal. Optimization of $\mathrm{CNC}$ end milling process parameters using PCA-based Taguchi method, International Journal of Engineering, Science and Technology, 2010, Vol. 2, No. 1, pp. 92-102.

20. Stone R.A. and Veevers A. The Taguchi influence on designed experiments, $J$ Chemometrics, 1994, 8: 103-110.

21. Houng J.Y., Liao J.H., Wu J.Y., Shen S.C. and Hsu H.F. Enhancement of asymmetric bioreduction of ethyl $4 \square$ chloro acetoacetate by the design of composition of culture medium and reaction conditions, Process Biochem, 2006, 42: $1 \square 7$.

22. Cobb B.D. and Clarkson J.M. A simple procedure for optimizing the polymerase chain reaction (PCR) using modified Taguchi methods, Nucleic Acids Res, 1994, 22: 3801-3805.

23. Han J.J., Yang T.H. and Rhee J.S. Optimization of reaction variables for sucrose monoester production using lipase in a solvent free system by taguchi's method, Biotechnol Tech, 1998, 12 (4): pp. $295 \square 299$.

24. Jeney C., Dobay O., Lengyel A., Adam E. and Nasz I. Taguchi optimization of ELISA procedures, $J$ Immun Method, 1999, 223: pp. 137-146.

25. Dasu V.V., Panda T. and Chidambaram M. Determination of significant parameters for improved griseofulvin production in a batch bioreactor by Taguchi's method, Process Biochem, 2003, 38: pp. 877 $\square 880$.

26. Krishna Prasad K., Venkata Mohan S., Sreenivas Rao R., Ranjan Pati B. and Sarma P.N. Laccase production by Pleurotus ostreatus 1804: Optimization of submerged 
B. Surapong et al., International Journal of Emerging Trends in Engineering Research, 9(7), July 2021, 929 - 936

culture conditions by Taguchi DOE methodology, Biochem Eng J, 2005, 24: pp.17 $\square 26$.

27. K. Siva Prasad, K.S.V.K. Sainath and B. Vijay Kumar. Investigation of material removal rate on mild steel-using taguchi technique, International Journal of Engineering Technology Science and Research, IJETSR, www.ijetsr.com ISSN 2394 - 3386, 2017, Volume 4, Issue 5, May.

28. Srinivas Athreya1 and Dr Y.D.Venkatesh. Application of taguchi method for optimization of process parameters in improving the surface roughness of lathe facing operation, International Refereed Journal of Engineering and Science (IRJES), 2012, pp. 13-19.

29. T. Foster. Basic Taguchi design of experiments, National Association of Industrial Technology Conference, Pittsburgh, PA. Ernest Doebelin.(No year specified). Engineering Experimentation, Tata MCGRAW HILL Publication, 2000.

30. Duan, Weiran, Dai, Yifan, Shu, Yong and Sherrington, Ian. Application of taguchi methods and ANOVA in optimization of process parameters for surface roughness of fused silica in the magnetorheological finishing processes, Advanced Materials Research, 2013, 662 (-). pp. 449-452. ISSN 1662-8985.

31. Hardeep Singh, Rajesh Khanna, M.P. Garg. Effect of cutting parameters on MRR and surface roughness in turning EN-8, International Journal of Current Engineering and Technology, 2011, Vol.1, No.1, pp.100-104.

32. Musunuru, et al. Optimisation of surface roughness and material removal rate in turning operation of Mild steel using taguchi method, International journal of engineering science \& research technology (IJESRT), (ISSN: 2277-9655), 2018, pp. 477-484. 